\title{
Empathy and Theory of Mind in Ultra-High Risk for Psychosis: Relations With Schizotypy and Executive Function
}

\author{
Wanji Kong ${ }^{1,2}$, Se Jun Koo ${ }^{2,3}$, Eunchong Seo ${ }^{1,2}$, Hye Yoon Park ${ }^{1,2}$, Eun Lee ${ }^{1,2}$, and Suk Kyoon An ${ }^{1,2,3 凶}$ \\ ${ }^{1}$ Department of Psychiatry, Yonsei University College of Medicine, Severance Hospital, Seoul, Republic of Korea \\ ${ }^{2}$ Section of Self, Affect and Neuroscience, Institute of Behavioral Science in Medicine, Yonsei University College of Medicine, Seoul, \\ Republic of Korea \\ ${ }^{3}$ Graduate Program in Cognitive Science, Yonsei University, Seoul, Republic of Korea
}

Objective While recent studies have found deficits in theory of mind (ToM) skills in individuals at ultra-high risk (UHR) for psychosis, empathic tendencies in these subjects remain unclear. The presence of high schizotypy and compromised executive functions, which are found in UHR individuals, would affect ToM skills and empathic tendencies. We investigated the ToM skills and empathic tendencies of UHR individuals and examined their relationship with schizotypy and executive function.

Methods This study included 28 UHR individuals and 28 age- and sex-matched healthy controls. All participants completed a self-reported empathic scale (Interpersonal Reactivity Index) and the Wisconsin Schizotypy Scales. Additionally, the ToM Picture Stories Task and Wisconsin Card Sorting Test were conducted.

Results UHR individuals showed a trend toward lower self-reported empathic tendencies; however, there were no differences in ToM skills between the two groups. Of the four subscales of the IRI, only empathic concern showed a significant difference between the two groups. Empathic concern was inversely associated with negative schizotypy.

Conclusion Our findings suggest that UHR individuals show relatively preserved cognitive empathy but compromised emotional empathy. Furthermore, in UHR individuals, the empathic concern subscale of the IRI was associated with negative schizotypy, while ToM skills were related to positive schizotypy and executive function. Psychiatry Investig 2021;18(11):1109-1116

Keywords Social cognition; Empathy; Theory of mind; Anhedonia; Magical ideation; Self-other boundary.

\section{INTRODUCTION}

Social cognitive deficits are a key feature of individuals with schizophrenia that affect daily functioning and outcomes. ${ }^{1,2}$ Theory of mind (ToM) and empathic tendencies are multidimensional domains of social cognition and have been used reciprocally without clear distinction in previous studies. ${ }^{3}$ ToM is the ability to infer others' mental states, including intentions and thoughts, and to attribute those states to others, while empathy describes the ability to react to one's own and other people's emotions. According to the empathy models of Shamay-

\footnotetext{
Received: March 30, 2021 Revised: July 10, 2021

Accepted: September 12, 2021

$\triangle$ Correspondence: Suk Kyoon An, MD, PhD

Department of Psychiatry, Yonsei University College of Medicine, 50-1 Yonsei-ro, Seodaemun-gu, 03722 Seoul, Republic of Korea

Tel: +82-2-2228-1585, Fax: +82-2-313-0891, E-mail: ansk@yuhs.ac

(c) This is an Open Access article distributed under the terms of the Creative Commons Attribution Non-Commercial License (https://creativecommons.org/licenses/bync/4.0) which permits unrestricted non-commercial use, distribution, and reproduction in any medium, provided the original work is properly cited.
}

Tsoory et al., ${ }^{4}$ both ToM and empathic tendencies have two distinct aspects: cognitive and affective. ${ }^{5,6}$ Cognitive ToM refers to the ability to infer others' beliefs, intentions, and thoughts, while affective ToM refers to the ability to think about others' feelings. Cognitive empathy is the ability to employ cognitive functions to take other people's perspectives, whereas emotional empathy is the ability to feel emotional reactions when observing other people's experiences. Thus, cognitive ToM is a prerequisite for affective ToM, and affective ToM is related to cognitive empathy, as well as emotional empathy to some extent. ${ }^{4}$

ToM abilities are empirically examined using verbal or visual behavioral tasks such as the Strange Stories Test, ${ }^{7}$ the Reading the Mind in the Eyes Test, ${ }^{8,9}$ and the ToM Picture Stories Task (ToM-PST).$^{10}$ Impairment of ToM in schizophrenia has been consistently reported. ${ }^{1,11}$ Unaffected first-degree relatives of schizophrenic patients ${ }^{12,13}$ and individuals at ultra-high risk (UHR) for psychosis $^{13}$ also show modest ToM deficits. Meanwhile, empathic tendencies are measured using self-reported questionnaires such as the Interpersonal Reactivity Index (IRI). ${ }^{14}$ 
Empathic tendencies in schizophrenic patients have mostly been investigated using the IRI, which consists of two cognitive (perspective taking and fantasy) and two emotional components (empathic concern and personal distress) of empathy. According to a previous meta-analysis, individuals with schizophrenia reported significant impairments in perspective taking (pooled effect size $[E S]=-0.53$ ), empathic concern (pooled $\mathrm{ES}=-0.28$ ), and fantasy (pooled $\mathrm{ES}=-0.19$ ), while experiencing more personal distress (pooled ES $=0.71$ ) ${ }^{15}$ Meanwhile, in one study of the IRI of patients with first-episode schizophrenia with subsequent meta-analytic comparisons with previous studies in patients with chronic schizophrenia, ${ }^{16,17}$ first-episode patients showed an increase in personal distress subscale scores at the trend-level while showing comparable levels in other subscales relative to those of normal controls. Moreover, this meta-analytic comparison found that there was a significant difference in perspective-taking and trend-level differences in fantasy between patients with first-episode schizophrenia and those with chronic schizophrenia. ${ }^{16,17}$ It was previously proposed that no impairment in perspective taking exists in patients with first-episode schizophrenia. ${ }^{16,17}$ In unaffected first-degree relatives of schizophrenic patients, no significant difference in IRI subscales was found compared to normal controls who did not have a family history of schizophrenia. ${ }^{12}$ However, little is known about self-reported empathy in UHR individuals. One study ${ }^{18}$ on behavioral assessments of empathic tendencies in individuals at UHR for psychosis showed that subjects who later developed overt psychosis were characterized by lower scores in emotional empathy but not in cognitive empathy.

It has been reported that social cognitive deficits are related to the level of schizotypy, executive function, duration of illness, use of psychiatric medications, co-morbidities, and sex. ${ }^{2,15,19-21}$ Schizotypy is the risk of psychosis that is continuously distributed in the general population. ${ }^{22-24}$ Schizotypy consists of three identifiable dimensions: 1) positive dimension, which is a cognitive-perceptual dimension that includes magical thinking, perceptual aberrations, or ideas of reference; 2) negative dimension, which is an interpersonal dimension and includes constricted affect, social anxiety, and social or physical anhedonia; and 3) disorganized dimension, which includes odd behavior or speech. ${ }^{25}$ Regarding the relationship between schizotypy and ToM, it was reported that only positive schizotypy negatively affects ToM performance in psychometrically-defined schizotypy groups. ${ }^{26}$ Regarding the relationship of schizotypy with both ToM and empathy, a structural equation modeling study in the general population demonstrated a significant negative relationship only between negative schizotypy and empathy as modeled by the empathic concern, perspective taking, and fantasy subscales of the IRI; however, there were no significant associations between each dimension of schizotypy and ToM. ${ }^{27}$
Executive function plays a role in social cognitive processes; deficits of executive function in schizophrenia may lead to poorer social cognitive processes. ${ }^{28-30}$ Executive function is defined as a group of neurocognitive processes that include mental set shifting, inhibition, planning, working memory, and cognitive flexibility. ${ }^{31}$ Regarding the relationship between executive function and ToM in UHR individuals, IQ was reported to be positively correlated with ToM performance; additionally, in UHR individuals, lower IQ $(<110)$ was associated with more prominent ToM disabilities when compared to higher IQ $(\geq 110) .{ }^{32,33}$ Regarding the relationship of executive function with empathic tendencies, a recent meta-analysis in a mixed sample of healthy volunteers and various clinical groups revealed that empathy, especially cognitive empathy, was positively related to executive function. ${ }^{34}$

The first aim of this study was to investigate the presence of impairment of self-reported empathic tendencies and ToM skills (measured by scores of the sequence and cognitive questionnaire of the ToM-PST ${ }^{10}$ ) in individuals at UHR for psychosis, who are known to show high levels of schizotypy ${ }^{35}$ and compromised executive function. ${ }^{36-38}$ The second aim was to examine the relationships of empathic tendencies and ToM skills with schizotypy (measured by anhedonia ${ }^{39}$ and magical ideation $^{40}$ ) and executive function (measured by the Wisconsin Card Sorting Test ${ }^{41}$ ) in UHR individuals. Based on previous findings, it was hypothesized that, in individuals at UHR for psychosis, both self-rated empathy and ToM skills would be impaired. In addition, we examined whether the domains of empathy and ToM could be associated with schizotypy and executive function in UHR individuals.

\section{METHODS}

\section{Participants}

Twenty-eight individuals (19 men and 9 women) at UHR for psychosis and 28 age- and sex-matched healthy controls (HC) participated in the present study. From July 2011 to November 2016, UHR participants were recruited from the research clinic FOR YOU at the Severance Hospital of the Yonsei University Health System in Seoul, Republic of Korea. Eligible participants were aged from 15 to 35 years and all of them were assessed using the Structured Clinical Interview for DSM-IV Axis I Disorders (SCID-IV). ${ }^{42,43}$ Individuals at UHR for psychosis were evaluated according to the Structured Interview for Prodromal Syndromes (SIPS). ${ }^{44}$ Each UHR participant met one or more of the following criteria based on the presence and severity of positive symptoms: 1) attenuated positive symptom syndrome (APSS, $n=25), 2$ ) brief intermittent psychotic syndrome (BIPS, $n=1$ ), and 3) genetic risk and deterioration syndrome (GRDS; $\mathrm{n}=7$ ). The DSM-IV diagnoses of the UHR 
group were as follows: depressive disorder $(n=14)$, anxiety disorder ( $\mathrm{n}=6$; social phobia, $\mathrm{n}=3$; obsessive-compulsive disorder, $\mathrm{n}=1$; panic disorder, $\mathrm{n}=1$; post-traumatic stress disorder, $\mathrm{n}=1$ ), somatoform disorder $(n=1)$, and depersonalization disorder $(n=1)$. Participants were excluded if they have a history of or currently have neurological disorders, traumatic brain injury, or intellectual disability (IQ $<70)$. This study was reviewed and approved by the Institutional Review Board of the Severance Hospital of the Yonsei University Health System (IRB No. 4-2014-0744). All individuals and parents of participants under 18 years of age provided written informed consent.

\section{Measures}

\section{Self-report assessment of empathic tendencies}

Empathic tendencies were assessed using the Interpersonal Reactivity Index (IRI), ${ }^{14,45}$ which is a 28 -item self-report questionnaire most widely used for assessing empathy as a multidimensional construct. The IRI consists of four subscales with 7 items under each: 1) empathic concern, the propensity to feel sympathy or compassion for others; 2) perspective taking, the propensity to adopt another's psychological point of view; 3 ) personal distress, the inclination to experience self-oriented negative feelings when seeing others in distress; and 4) fantasy, the inclination to transpose oneself into feelings and actions of fictitious characters in imaginary situations such as books or movies. The empathic concern and personal distress subscales represent affective aspects, whereas the perspective-taking and fantasy subscales represent the cognitive aspects of empathy. The total score for each subscale ranges from 0 to 28 . In the present study, the internal consistency of each subscale (Cronbach's alpha) ranged from 0.71 to 0.79 .

\section{Behavioral measurement of ToM skills}

ToM skills were evaluated using the ToM Picture Stories Task (ToM-PST) ${ }^{10}$ which consists of six cartoon picture sets with four pictures each. The test was carried out in two steps. In the first step, participants were asked to rearrange the shuffled cards into a logical sequence of events. For each trial, participants scored 2 points if the first and last cards were in the correct order and one point each for correctly ordering the second and third cards (ToM-PST, sequence: $0-36$ points). In the second step, after the cards were sequenced correctly by the participant or by an examiner, the participant was requested to describe each picture and infer the cognitive and affective mental states of the characters. Cognitive questions were directed towards the false-beliefs and intentions of the cartoon characters, whereas affective questions asked about the emotions and feelings of the characters. ${ }^{46}$ In the present study, only the cognitive questionnaires were used since the affective ques- tionnaires were not available at the author's lab at the start of the study. A point was given in the ToM-PST cognitive questionnaire for logical reasoning and comprehension of first-, second-, and third-order false beliefs (cognitive questionnaires: 0-23 points). There were two missing data for each group.

\section{Assessment of self-reported schizotypy}

Psychometric schizotypy was assessed using the self-reported Wisconsin Schizotypy Scales, ${ }^{47}$ specifically, the Magical Ideation Scale ${ }^{40}$ and the Revised Social Anhedonia Scale. ${ }^{39}$ The Magical Ideation Scale is a 30-item true-false scale that assesses unusual beliefs of tendency of causation that are invalid by conventional standards. This scale contains statements that are mainly related to unusual associations between mental processes and external events, occult or fantastic ideas, superstitions, and déjà vu, and also contains a few statements related to delusion-like phenomena such as ideas of reference. The Revised Social Anhedonia Scale assesses anhedonia in hypothetical emotional experiences and consists of 40 true-false items that measure deficits in the ability to experience pleasure from non-physical stimuli such as interpersonal relationships. The Magical Ideation Scale represents the positive aspect of schizotypy, whereas the Revised Social Anhedonia scale represents the negative aspect of schizotypy. We used the Korean version of the Magical Ideation Scale and Social Anhedonia Scale, which is widely used in Korean psychiatric research. ${ }^{35,48,49}$ In the present study, the internal consistency (Cronbach's alpha) of the Magical Ideation Scale was 0.76, and that of the Revised Social Anhedonia Scale was 0.93 .

\section{Assessment of executive function}

The Wisconsin Card Sorting Test ${ }^{41}$ was conducted to assess executive function; the total number of errors was used in this study. There was one missing data point in the UHR group.

\section{Statistical analysis}

Independent t-tests and $\chi^{2}$ tests were conducted to analyze the differences in demographic and clinical characteristics between the two groups. The effects of being at UHR for psychosis, sex, and their interactions on IRI and ToM-PST, were tested separately using a multivariate analysis of variance (MANOVA). Post-hoc comparisons between the HC and UHR groups were conducted using one-way analysis of variance (ANOVA) for each IRI and ToM-PST score. In the UHR group, stepwise multiple linear regression analyses were performed to examine the relationships between IRI and ToM-PST with schizotypy and executive function. Each subscale of the IRI and the ToM-PST was analyzed as dependent variables, while the scores from the Magical Ideation Scale and Revised Social Anhedonia Scale, as well as the total errors in WCST, were treated as inde- 
pendent variables. Statistical analyses were performed using Statistical Package for the Social Sciences (SPSS 25; IBM Corp., Armonk, NY, USA). The level of significance was defined as a p-value less than 0.05 , and a p-value between 0.05 and 0.10 was considered a trend level of significance.

\section{RESULTS}

\section{Demographic and clinical characteristics}

The demographic and clinical characteristics of all participants are shown in Table 1. There were no significant differences in age, sex, total duration of education, or total errors in
WCST between the two groups. The UHR group demonstrated higher scores on the Revised Social Anhedonia scale, but there was no difference in the Magical Ideation Scale between the two groups.

\section{Effect of UHR for psychosis and sex on the empathic tendencies and the ToM skills}

For empathic tendencies (Table 2), significant effects at the trend level were observed with the groups [HC vs. UHR; F $(4,49)=$ 2.3, $\mathrm{p}=0.070$ ] and sex [male vs. female; $\mathrm{F}(4,49)=2.1, \mathrm{p}=0.097$ ]. There were no significant interaction effects between the groups and sex $[\mathrm{F}(2,47)=1.0, \mathrm{p}=0.442]$. Post-hoc ANOVAs to compare

Table 1. Demographic and clinical characteristics of healthy controls and individuals at ultra-high risk for psychosis

\begin{tabular}{|c|c|c|c|}
\hline & $\mathrm{HC}(\mathrm{N}=28)$ & $\operatorname{UHR}(\mathrm{N}=28)$ & Statistical parameters \\
\hline Age (mean years $\pm S D)$ & $20.4 \pm 2.9$ & $20.3 \pm 3.4$ & $\mathrm{t}=0.09, \mathrm{df}=54, \mathrm{p}=0.932$ \\
\hline Sex (male/female) & $19 / 9$ & $19 / 9$ & \\
\hline Education (mean years $\pm S D$ ) & $13.6 \pm 1.9$ & $13.1 \pm 1.7$ & $\mathrm{t}=1.12, \mathrm{df}=54, \mathrm{p}=0.268$ \\
\hline \multicolumn{4}{|l|}{ PANSS* } \\
\hline Positive scale & $7.1 \pm 0.5$ & $13.0 \pm 3.9$ & $\mathrm{t}=-7.70, \mathrm{df}=52, \mathrm{p}<0.001$ \\
\hline Negative scale & $7.1 \pm 0.5$ & $17.4 \pm 6.1$ & $\mathrm{t}=-8.76, \mathrm{df}=52, \mathrm{p}<0.001$ \\
\hline General psychopathology scale & $16.2 \pm 0.6$ & $34.2 \pm 5.7$ & $\mathrm{t}=-16.40, \mathrm{df}=52, \mathrm{p}<0.001$ \\
\hline \multicolumn{4}{|l|}{ Antipsychotic medications } \\
\hline Naïve/medicated & & $21 / 7$ & \\
\hline Chlorpromazine equivalent dose (mg/d) & & $99.6(90.5)$ & \\
\hline \multicolumn{4}{|l|}{ Self-reported schizotypy } \\
\hline Magical Ideation Scale & $8.7 \pm 4.2$ & $8.1 \pm 5.9$ & $\mathrm{t}=0.40, \mathrm{df}=54, \mathrm{p}=0.692$ \\
\hline Revised Social Anhedonia Scale & $7.7 \pm 4.1$ & $22.0 \pm 8.3$ & $\mathrm{t}=-8.16, \mathrm{df}=54, \mathrm{p}<0.001$ \\
\hline \multicolumn{4}{|l|}{ Executive function } \\
\hline Total errors of Wisconsin Card Sorting Test $^{\dagger}$ & $26.1 \pm 17.4$ & $19.0 \pm 15.8$ & $\mathrm{t}=1.58, \mathrm{df}=53, \mathrm{p}=0.119$ \\
\hline
\end{tabular}

${ }^{*} 1$ missing data in each group; ${ }^{\dagger} 1$ missing data in UHR group. HC, healthy controls; UHR, ultra-high risk; PANSS, Positive and Negative Syndrome Scale; SD, standard deviation

Table 2. Empathic tendencies and theory of mind skills of healthy controls and individuals at ultra-high risk for psychosis: MANOVAs and post hoc ANOVAs with subscales of the IRI and ToM-PST as dependent variables

\begin{tabular}{lccc}
\hline & Group & Sex & Group $\times$ sex \\
\hline $\begin{array}{l}\text { MANOVA } \\
\text { Post hoc ANOVA }\end{array}$ & $\mathrm{F}(4,49)=2.319, \mathrm{p}=0.070$ & $\mathrm{~F}(4,49)=2.087, \mathrm{p}=0.097$ & $\mathrm{~F}(4,49)=0.953, \mathrm{p}=0.442$ \\
$\quad$ Empathic concern of IRI & $\mathrm{F}(1,52)=4.980, \mathrm{p}=0.030$ & $\mathrm{~F}(1,52)=0.027, \mathrm{p}=0.871$ & $\mathrm{~F}(1,52)=0.345, \mathrm{p}=0.560$ \\
$\quad$ Perspective taking of IRI & $\mathrm{F}(1,52)=1.510, \mathrm{p}=0.225$ & $\mathrm{~F}(1,52)=6.569, \mathrm{p}=0.013$ & $\mathrm{~F}(1,52)=3.198, \mathrm{p}=0.080$ \\
$\quad$ Personal distress of IRI & $\mathrm{F}(1,52)=2.141, \mathrm{p}=0.149$ & $\mathrm{~F}(1,52)=0.421, \mathrm{p}=0.520$ & $\mathrm{~F}(1,52)=0.097, \mathrm{p}=0.757$ \\
$\quad$ Fantasy of IRI & $\mathrm{F}(1,52)=0.083, \mathrm{p}=0.774$ & $\mathrm{~F}(1,52)=0.624, \mathrm{p}=0.433$ & $\mathrm{~F}(1,52)=0.257, \mathrm{p}=0.614$ \\
MANOVA & $\mathrm{F}(2,47)=2.378, \mathrm{p}=0.104$ & $\mathrm{~F}(2,47)=2.225, \mathrm{p}=0.119$ & $\mathrm{~F}(2,47)=0.512, \mathrm{p}=0.602$ \\
Post hoc ANOVA & & & $\mathrm{F}(1,48)=0.817, \mathrm{p}=0.371$ \\
$\quad \begin{array}{l}\text { ToM-PST sequence } \\
\text { ToM-PST cognitive questionnaire }\end{array}$ & $\mathrm{F}(1,48)=3.114, \mathrm{p}=0.084$ & $\mathrm{~F}(1,48)=4.284, \mathrm{p}=0.044$ & $\mathrm{~F}(1,48)=0.845, \mathrm{p}=0.363$ \\
\hline
\end{tabular}

MANOVA, multivariate analysis of variance; ANOVA, analysis of variance; IRI, interpersonal reactivity index; ToM-PST, theory of mind picture stories task 
IRI subscales between UHR individuals and HC, as well as between males and females, were conducted since the main effects of group and sex were at the trend level. Empathic concern (14.5 \pm 5.1$)$ in UHR individuals was significantly lower than that $(18.0 \pm 4.6)$ in $\mathrm{HC}[\mathrm{F}(1,52)=5.0, \mathrm{p}=0.030]$. Perspectivetaking $(13.6 \pm 4.6)$ in women was significantly lower than that $(16.9 \pm 4.8)$ in men $[F(1,52)=6.6, p=0.013]$. There were no significant effects.

For ToM skills (Table 2), there were no significant or trendlevel effects by group $[\mathrm{F}(2,47)=2.4, \mathrm{p}=0.104]$ or $\operatorname{sex}[\mathrm{F}(2,47)=$ $2.2, \mathrm{p}=0.119]$; furthermore, there were no interaction effects between group and $\operatorname{sex}[\mathrm{F}(2,47)=0.5, \mathrm{p}=0.602]$.

\section{Associations of empathic tendencies and ToM skills with schizotypy and executive function in individuals at UHR for psychosis}

Stepwise multiple linear regression analyses were conducted using the Magical Ideation Scale, Revised Social Anhedonia Scale, and total errors in WCST as independent variables. Empathic concern $\left[\mathrm{F}(1,25)=5.2, \mathrm{p}=0.032\right.$, adj. $\left.\mathrm{R}^{2}=0.14\right]$ and perspective-taking $\left[\mathrm{F}(1,25)=6.5, \mathrm{p}=0.017\right.$, adj. $\left.\mathrm{R}^{2}=0.18\right]$ were negatively associated with social anhedonia. Personal distress and fantasy were positively related to magical ideation [personal distress: $\mathrm{F}(1,25)=4.4, \mathrm{p}=0.047$, adj. $\mathrm{R}^{2}=0.12$; fantasy: $\mathrm{F}(1,25)=$ $7.6, \mathrm{p}=0.011$, adj. $\mathrm{R}^{2}=0.20$ ]. For ToM skills, the ToM-PST sequence was negatively associated with total errors in WCST $(\mathrm{p}=0.001)$ and magical ideation $(\mathrm{p}=0.007)[\mathrm{F}(2,22)=10.9, \mathrm{p}=$ 0.001 , adj. $\left.\mathrm{R}^{2}=0.45\right]$. The ToM-PST cognitive questionnaire was also negatively related to the total errors in WCST $(\mathrm{p}=0.012)$ and magical ideation $(\mathrm{p}=0.084)[\mathrm{F}(2,22)=5.2, \mathrm{p}=0.014$, adj. $\left.\mathrm{R}^{2}=0.26\right]$. All variance inflation factor $(\mathrm{VIF}) \mathrm{s}$ were less than 1.1 (Table 3).

\section{DISCUSSION}

This study aimed to investigate whether empathic tendencies and ToM skills are impaired in individuals at UHR for psychosis and to examine their relationships with schizotypy and executive function. There was a trend-level difference in self-reported empathic tendencies but there was no difference in the ToM skills between the two groups. The compromised empathic concern of the IRI contributed to the group differences. Empathic concern was solely associated with negative schizotypy, while ToM skills were related to positive schizotypy and executive function in UHR individuals.

\section{Empathic tendencies in individuals at UHR for psychosis}

The empathic concern subscale, but not the cognitive empathy subscale, of IRI was found to be compromised in UHR individuals. These results are consistent with the findings of a behaviorally-assessed empathy study which showed that, while emotional empathy in UHR individuals who later developed overt psychosis was poorer compared to HC, cognitive empathy was relatively preserved. ${ }^{18}$ Regarding the emotional components of empathic tendency, the impairment of empathic concern in individuals at UHR for psychosis is consistent with our previous finding of reduced current pleasurable response to emotional stimuli in a laboratory setting in UHR individuals. ${ }^{50}$ The finding of reduced empathic concern without an increase in personal distress in UHR individuals is inconsistent with previous IRI findings in patients with first-episode schizophrenia that showed intact empathic concern and increased personal distress. ${ }^{16,17}$ Regarding cognitive empathy, the relatively intact cognitive empathic tendencies in UHR individuals is consistent with and supports the previous IRI findings

Table 3. Stepwise multiple linear regression analyses of empathic tendencies and theory of mind skills with schizotypy and executive function of individuals at ultra-high risk for psychosis

\begin{tabular}{|c|c|c|c|c|c|c|c|}
\hline Dependent variables & Independent variables & B & $\mathrm{SE}$ & $\beta$ & $\mathrm{t}$ & $\mathrm{p}$ & Model properties \\
\hline \multicolumn{8}{|l|}{ Empathic tendencies } \\
\hline Empathic concern of IRI & Revised social anhedonia & -0.24 & 0.11 & -0.42 & -2.29 & 0.032 & $\mathrm{~F}_{1,25}=5.2, \mathrm{p}=0.032, \mathrm{R}^{2}=0.17$, adj. $\mathrm{R}^{2}=0.14$ \\
\hline Perspective taking of IRI & Revised social anhedonia & -0.26 & 0.10 & -0.45 & -2.55 & 0.017 & $\mathrm{~F}_{1,25}=6.5, \mathrm{p}=0.017, \mathrm{R}^{2}=0.21$, adj. $\mathrm{R}^{2}=0.18$ \\
\hline Personal distress of IRI & Magical ideation & 0.37 & 0.18 & 0.39 & 2.09 & 0.047 & $\mathrm{~F}_{1,25}=4.4, \mathrm{p}=0.047, \mathrm{R}^{2}=0.15$, adj. $\mathrm{R}^{2}=0.12$ \\
\hline Fantasy of IRI & Magical ideation & 0.48 & 0.17 & 0.48 & 2.75 & 0.011 & $\mathrm{~F}_{1,25}=7.6, \mathrm{p}=0.011, \mathrm{R}^{2}=0.23$, adj. $\mathrm{R}^{2}=0.20$ \\
\hline \multicolumn{8}{|l|}{ Theory of mind skills } \\
\hline \multirow[t]{2}{*}{ ToM-PST sequence } & Total errors of WCST & -0.16 & 0.05 & -0.56 & -3.68 & 0.001 & $\mathrm{~F}_{2,22}=10.9, \mathrm{p}=0.001, \mathrm{R}^{2}=0.50$, adj. $\mathrm{R}^{2}=0.45$ \\
\hline & Magical ideation & -0.36 & 0.12 & -0.45 & -2.98 & 0.007 & \\
\hline \multirow[t]{2}{*}{ ToM-PST cognitive questionnaire } & Total errors of WCST & -0.10 & 0.04 & -0.48 & -2.73 & 0.012 & $\mathrm{~F}_{2,22}=5.2, \mathrm{p}=0.014, \mathrm{R}^{2}=0.32$, adj. $\mathrm{R}^{2}=0.26$ \\
\hline & Magical ideation & -0.18 & 0.10 & -0.32 & -1.81 & 0.084 & \\
\hline
\end{tabular}

All variance inflation factor (VIF)s were less than 1.1. B, unstandardized coefficient; SE, standard error of unstandardized coefficient; $\beta$, standardized coefficient; adj. $\mathrm{R}^{2}$, adjusted R-square; IRI, interpersonal reactivity index; ToM-PST, theory of mind picture stories task; WCST, Wisconsin Card Sorting Test 
of intact perspective taking and fantasy in patients with firstepisode schizophrenia. ${ }^{16,17}$ Meanwhile, a previous meta-analysis of patients with chronic schizophrenia revealed impaired perspective taking in these patients. ${ }^{15}$ Therefore, emotional and cognitive empathic tendencies as a whole may evolve differently depending on the phase and progression of a patient's illness.

\section{ToM skills in individuals at UHR for psychosis}

The overall performance of ToM skills in UHR individuals did not differ significantly from that of $\mathrm{HC}$. This finding seems to be incompatible with a previous meta-analysis ${ }^{51}$ that reported ToM impairment on verbal ToM tasks in UHR individuals; furthermore, this finding is also incompatible with previous studies that consistently demonstrated impaired ToM performance on false belief tasks in patients with schizophrenia. ${ }^{11,13}$ However, previous studies on UHR individuals using false belief tasks as a measure of ToM have shown mixed results. ${ }^{33,52-54}$ One study, which divided participants into high- $(\geq 110)$ and low-IQ groups, revealed that the UHR group with high IQ performed just as well as $\mathrm{HC}$ with high IQ on ToM tasks. ${ }^{33}$ Therefore, in this study, the results of the ToM-PST could be partly due to the comparable level of performance in the Wisconsin Card Sorting Test in both groups.

\section{Relationship of empathic tendencies and ToM skills with schizotypy and executive function}

The empathic concern was exclusively associated with negative schizotypy but not with executive function. Our findings are consistent with a previous study on behaviorally assessed empathy of UHR individuals who later developed overt psychosis; in that study, it was shown that decreased emotional empathy was related to negative symptoms. ${ }^{18}$ In addition, this finding is consistent with previous studies that showed a negative correlation between emotional empathy and negative schizotypy in schizophrenic patient ${ }^{55}$ and non-clinical participants..$^{27,56,57}$ Since social anhedonia, which is a negative schizotypy, is defined as a decrease in pleasure derived from interpersonal sources, decreased empathic concern in UHR individuals may reflect indifference and lower emotional reactivity to other people's experiences. ${ }^{39,58}$

ToM skills were found to be related to both positive schizotypy and executive function in UHR individuals. These findings are consistent with previous findings that showed ToM impairment in the positive schizotypy group but not in the negative group. ${ }^{26}$ Since magical ideation is an unusual belief in forms of causation that are invalid by conventional standards and unusual associations between mental processes and external events, ${ }^{40} \mathrm{UHR}$ individuals with increased magical ideation may not be able to discriminate between their own mental state and that of others; thus, they cannot properly perform the false-belief test. A previous meta-analysis of the general population showed a significant association between schizotypy and poorer ToM performance and demonstrated that various aspects of ToM skills are affected differently by different schizotypy dimensions; furthermore, the researchers suggested that neurocognition might be a potential mediator. ${ }^{2}$ As previously demonstrated in schizophrenic patients ${ }^{11}$ and in individuals at UHR for psychosis, ${ }^{33,52,53}$ ToM skills, but not empathic concern, were shown to be associated with executive function in these individuals. Measurements of ToM skills examine the extent to which a person can match with another person's mental state or emotion; that is, the ability to see from another person's perspective while maintaining one's own stance. However, empathic tendencies that are measured by a self-reported scale may only reflect how much effort one can exert to empathize with others or subjectively experience empathic emotions under given situations. Therefore, the discrepancy in the association of executive function with empathic tendencies and ToM skills in this study might be attributed to differences in the underlying constructs of ToM skills and empathic tendencies. Another possible explanation of this differential relationship of two measures of social cognition with executive function is derived from the methodological differences in evaluating ToM skills and empathic tendencies; that is, the sequencing ability of ToM-PST may also reflect executive function, while self-reported empathic tendencies may be not dependent on executive function. Thus, this differential relationship could just reflect a methodological characteristic of our measures.

Perspective taking is related to social anhedonia. Perspective taking assesses the propensity to adopt another's psychological point of view in an effort to understand and consider the feelings of others. Thus, anhedonia, which is a negative schizotypy, may act as a hindrance to cognitive empathy. Furthermore, it was found that fantasy and personal distress are correlated with magical ideation in UHR individuals. Subjects at UHR for psychosis who have higher levels of magical ideation may not attribute the source of mental states to others and thus show increased empathic tendency to place themselves into hypothetical positions and to direct the distressful emotional states of other people toward themselves.

This study has several limitations. First, the relatively small sample size and the predominance of the male sex in the participants may have influenced the study; thus, the ToM skills of the UHR individuals in this study were not significantly different from those of normal controls. Second, this study was cross-sectional in design; thus, the causal relations of empathy and ToM with schizotypy and executive function cannot be determined. Third, behavioral performances of affective ToM were not examined because the affective questionnaires of ToM- 
PST were not available prior to the start of this study. Recent studies found discrepancies in empathy when using behavioral or self-report measurements in schizophrenic patients as these individuals tend to overestimate their empathy compared to what is observed by their caregivers. ${ }^{59}$ Therefore, future longitudinal follow-up studies should include behavioral measurements of empathy and comprehensive ToM assessments to elucidate the exact picture of social cognition in UHR individuals.

In conclusion, our findings suggest that individuals at UHR for psychosis experience reduced empathic concern compared to healthy controls, despite their preserved cognitive empathic tendencies and ToM skills. This reduced tendency for empathic concern may be related to stronger negative schizotypy rather than neurocognitive function in UHR individuals.

\section{Availability of Data and Material}

The datasets generated or analyzed during the study are available from the corresponding author on reasonable request.

\section{Conflicts of Interest}

The authors have no potential conflicts of interest to disclose.

\section{Author Contributions}

Conceptualization: Suk Kyoon An. Data curation: all authors. Formal analysis: Wanji Kong, Se Jun Koo, Suk Kyoon An. Funding acquisition: Eun Lee, Suk Kyoon An. Investigation: all authors. Methodology: Wanji Kong, Se Jun Koo. Project administration: Eun Lee, Suk Kyoon An. Resources: Eun Lee, Suk Kyoon An. Software: Wanji Kong, Se Jun Koo. Supervision: Suk Kyoon An. Valildation: Suk Kyoon An. Visualization: Wanji Kong. Writing—original draft: Wanji Kong. Writing—review \& editing: all authors.

\section{ORCID iDs}

Wanji Kong

Se Jun Koo

Eunchong Seo

Hye Yoon Park

Eun Lee

Suk Kyoon An

https://orcid.org/0000-0002-9843-4178 https://orcid.org/0000-0002-9051-8684 https://orcid.org/0000-0003-2644-266X https://orcid.org/0000-0001-9579-8112 https://orcid.org/0000-0002-7462-0144 https://orcid.org/0000-0003-4576-6184

\section{Funding Statement}

This work was supported by the Basic Science Research Program through the National Research Foundation of Korea funded by the Ministry of Science, ICT \& Future Planning, Republic of Korea (Grant number: 2017R1A2B3008214).

\section{REFERENCES}

1. Green MF, Horan WP, Lee J. Social cognition in schizophrenia. Nat Rev Neurosci 2015;16:620-631.

2. Bora E. Theory of mind and schizotypy: a meta-analysis. Schizophr Res 2020;222:97-103.

3. Quesque F, Rossetti Y. What do theory-of-mind tasks actually measure? Theory and practice. Perspect Psychol Sci 2020;15:384-396.

4. Shamay-Tsoory SG, Harari H, Aharon-Peretz J, Levkovitz Y. The role of the orbitofrontal cortex in affective theory of mind deficits in criminal offenders with psychopathic tendencies. Cortex 2010;46:668-677.

5. Bensalah L, Caillies S, Anduze M. Links among cognitive empathy, theory of mind, and affective perspective taking by young children. J Genet Psychol 2016;177:17-31.

6. Sebastian CL, Fontaine NM, Bird G, Blakemore SJ, Brito SA, McCrory
EJ, et al. Neural processing associated with cognitive and affective theory of mind in adolescents and adults. Soc Cogn Affect Neurosci 2012; 7:53-63.

7. Happé FG. An advanced test of theory of mind: understanding of story characters' thoughts and feelings by able autistic, mentally handicapped, and normal children and adults. J Autism Dev Disord 1994;24:129-154.

8. Baron-Cohen S, Wheelwright S, Hill J, Raste Y, Plumb I. The "Reading the Mind in the Eyes" Test revised version: a study with normal adults, and adults with Asperger syndrome or high-functioning autism. J Child Psychol Psychiatry 2001;42:241-251.

9. Koo SJ, Kim YJ, Han JH, Seo E, Park HY, Bang M, et al. "Reading the Mind in the Eyes Test": translated and Korean versions. Psychiatry Investig 2021;18:295-303.

10. Brüne M. Emotion recognition, 'theory of mind,' and social behavior in schizophrenia. Psychiatry Res 2005;133:135-147.

11. Bora E, Yucel M, Pantelis C. Theory of mind impairment in schizophrenia: meta-analysis. Schizophr Res 2009;109:1-9.

12. Montag C, Neuhaus K, Lehmann A, Krüger K, Dziobek I, Heekeren $\mathrm{HR}$, et al. Subtle deficits of cognitive theory of mind in unaffected firstdegree relatives of schizophrenia patients. Eur Arch Psychiatry Clin Neurosci 2012;262:217-226.

13. Bora E, Pantelis C. Theory of mind impairments in first-episode psychosis, individuals at ultra-high risk for psychosis and in first-degree relatives of schizophrenia: systematic review and meta-analysis. Schizophr Res 2013;144:31-36

14. Davis MH. Measuring individual differences in empathy: evidence for a multidimensional approach. J Pers Soc Psychol 1983;44:113-126.

15. Bonfils KA, Lysaker PH, Minor KS, Salyers MP. Empathy in schizophrenia: a meta-analysis of the Interpersonal Reactivity Index. Psychiatry Res 2017;249:293-303.

16. Achim AM, Ouellet R, Roy MA, Jackson PL. Assessment of empathy in first-episode psychosis and meta-analytic comparison with previous studies in schizophrenia. Psychiatry Res 2011;190:3-8.

17. Montag C, Heinz A, Kunz D, Gallinat J. Self-reported empathic abilities in schizophrenia. Schizophr Res 2007;92:85-89.

18. Montag C, Brandt L, Lehmann A, De Millas W, Falkai P, Gaebel W, et al. Cognitive and emotional empathy in individuals at clinical high risk of psychosis. Acta Psychiatr Scand 2020;142:40-51.

19. Thibaudeau É, Achim AM, Parent C, Turcotte M, Cellard C. A metaanalysis of the associations between theory of mind and neurocognition in schizophrenia. Schizophr Res 2020;216:118-128.

20. Oliveri LN, Awerbuch AW, Jarskog LF, Penn DL, Pinkham A, Harvey PD. Depression predicts self assessment of social function in both patients with schizophrenia and healthy people. Psychiatry Res 2020;284: 112681.

21. Mathersul D, Palmer DM, Gur RC, Gur RE, Cooper N, Gordon E, et al. Explicit identification and implicit recognition of facial emotions: II. Core domains and relationships with general cognition. J Clin Exp Neuropsychol 2009;31:278-291.

22. Grant P, Green MJ, Mason OJ. Models of schizotypy: the importance of conceptual clarity. Schizophr Bull 2018;44:S556-S563.

23. Meehl PE. Schizotaxia, schizotypy, schizophrenia. Am Psychol 1962;17: 827-838.

24. Meehl PE. Toward an integrated theory of schizotaxia, schizotypy, and schizophrenia. J Pers Disord 1990;4:1-99.

25. Nelson MT, Seal ML, Pantelis C, Phillips LJ. Evidence of a dimensional relationship between schizotypy and schizophrenia: a systematic review. Neurosci Biobehav Rev 2013;37:317-327.

26. Gooding DC, Pflum MJ. Theory of Mind and psychometric schizotypy. Psychiatry Res 2011;188:217-223.

27. Bedwell JS, Compton MT, Jentsch FG, Deptula AE, Goulding SM, Tone EB. Latent factor modeling of four schizotypy dimensions with theory of mind and empathy. PLoS One 2014;9:e113853.

28. Perner J, Lang B. Development of theory of mind and executive control. Trends Cogn Sci 1999;3:337-344. 
29. Langdon R, Coltheart M, Ward PB, Catts SV. Mentalising, executive planning and disengagement in schizophrenia. Cogn Neuropsychiatr 2001;6:81-108.

30. Champagne-Lavau M, Charest A, Anselmo K, Rodriguez JP, Blouin G. Theory of mind and context processing in schizophrenia: the role of cognitive flexibility. Psychiatry Res 2012;200:184-192.

31. Miyake A, Friedman NP, Emerson MJ, Witzki AH, Howerter A, Wager TD. The unity and diversity of executive functions and their contributions to complex "Frontal Lobe" tasks: a latent variable analysis. Cogn Psychol 2000;41:49-100.

32. Barbato M, Liu L, Cadenhead KS, Cannon TD, Cornblatt BA, McGlashan TH, et al. Theory of mind, emotion recognition and social perception in individuals at clinical high risk for psychosis: Findings from the NAPLS-2 cohort. Schizophr Res Cogn 2015;2:133-139.

33. Hur JW, Byun MS, Shin NY, Shin YS, Kim SN, Jang JH, et al. General intellectual functioning as a buffer against theory-of-mind deficits in individuals at ultra-high risk for psychosis. Schizophr Res 2013;149: 83-87.

34. Yan Z, Hong S, Liu F, Su Y. A meta-analysis of the relationship between empathy and executive function. Psych J 2020;9:34-43.

35. Bang M, Park JY, Kim KR, Lee SY, Song YY, Kang JI, et al. Psychotic conversion of individuals at ultra-high risk for psychosis: The potential roles of schizotypy and basic symptoms. Early Interv Psychiatry 2019; 13:546-554.

36. Carrión RE, Goldberg TE, McLaughlin D, Auther AM, Correll CU, Cornblatt BA. Impact of neurocognition on social and role functioning in individuals at clinical high risk for psychosis. Am J Psychiatry 2011;168:806-813.

37. Zheng W, Zhang QE, Cai DB, Ng CH, Ungvari GS, Ning YP, et al. Neurocognitive dysfunction in subjects at clinical high risk for psychosis: a meta-analysis. J Psychiatr Res 2018;103:38-45.

38. Bang M, Kim KR, Song YY, Baek S, Lee E, An SK. Neurocognitive impairments in individuals at ultra-high risk for psychosis: who will really convert? Aust N Z J Psychiatry 2015;49:462-470.

39. Chapman LJ, Chapman JP, Raulin ML. Scales for physical and social anhedonia. J Abnorm Psychol 1976;85:374-382.

40. Eckblad M, Chapman LJ. Magical ideation as an indicator of schizotypy. J Consult Clin Psychol 1983;51:215-225.

41. Heaton RK, Chelune GJ, Talley JL, Kay GG, Curtis G. Wisconsin Card Sorting Test Manual: Revised and Expanded. Odessa (FL): Psychological Assessment Resources, Inc; 1993.

42. First MB, Gibbon M, Spitzer RL, Williams JBW. Structured Clinical Interview for DSM-IV Axis I Disorders: Non-Patients Edition (SCIDI/ PS), Version 2. New York: New York State Psychiatric Institute Biometric Research; 1996.

43. First MB, Spitzer RL, Gibbon M, Williams JBW. Structured Clinical Interview for DSM-IV Axis I Disorders: Patient Edition (SCID-I/P), Version 2. New York: New York State Psychiatric Institute Biometric Research; 1996.

44. McGlashan T, Miller T, Woods S, Rosen J, Hoffman R, Davidson L.
Structured Interview for Prodromal Syndromes. New Haven, CT: PRIME Research Clinic, Yale School of Medicine; 2001.

45. Kang I, Kee S, Kim SE, Jeong B, Hwang JH, Song JE, et al. Reliability and validity of the Korean-version of Interpersonal Reactivity Index. J Korean Neuropsychiatr Assoc 2009;48:352-358.

46. Brüne M, Abdel-Hamid M, Lehmkämper C, Sonntag C. Mental state attribution, neurocognitive functioning, and psychopathology: what predicts poor social competence in schizophrenia best? Schizophr Res 2007;92:151-159.

47. Chapman LJ, Chapman JP, Kwapil TR, Eckblad M, Zinser MC. Putatively psychosis-prone subjects 10 years later. J Abnorm Psychol 1994; 103:171-183.

48. Seo E, Bang M, Lee E, An SK. Aberrant tendency of noncurrent emotional experiences in individuals at ultra-high risk for psychosis. Psychiatry Investig 2018;15:876-883.

49. Kim JJ. Comparison of the characteristics of anhedonia between patients with schizophrenia and depressive disorder. J Korean Neuropsychiatr Assoc 2010;49:570-577.

50. Jhung K, Park JY, Song YY, Kang JI, Lee E, An SK. Experiential pleasure deficits in the prodrome: a study of emotional experiences in individuals at ultra-high risk for psychosis and recent-onset schizophrenia. Compr Psychiatry 2016;68:209-216.

51. van Donkersgoed RJ, Wunderink L, Nieboer R, Aleman A, Pijnenborg GH. Social cognition in individuals at ultra-high risk for psychosis: a meta-analysis. PLoS One 2015;10:e0141075.

52. Stanford AD, Messinger J, Malaspina D, Corcoran CM. Theory of mind in patients at clinical high risk for psychosis. Schizophr Res 2011;131: 11-17.

53. Chung YS, Kang DH, Shin NY, Yoo SY, Kwon JS. Deficit of theory of mind in individuals at ultra-high-risk for schizophrenia. Schizophr Res 2008;99:111-118.

54. Ohmuro N, Katsura M, Obara C, Kikuchi T, Sakuma A, Iizuka K, et al. Deficits of cognitive theory of mind and its relationship with functioning in individuals with an at-risk mental state and first-episode psychosis. Psychiatry Res 2016;243:318-325.

55. Berger P, Bitsch F, Jakobi B, Nagels A, Straube B, Falkenberg I. Cognitive and emotional empathy in patients with schizophrenia spectrum disorders: a replication and extension study. Psychiatry Res 2019;276: 56-59.

56. Wang Y, Liu WH, Li Z, Wei XH, Jiang XQ, Neumann DL, et al. Dimensional schizotypy and social cognition: an fMRI imaging study. Front Behav Neurosci 2015;9:133.

57. Yang HX, Shi HS, Ni K, Wang Y, Cheung EFC, Chan RCK. Exploring the links between alexithymia, empathy and schizotypy in college students using network analysis. Cogn Neuropsychiatry 2020;25:245-253.

58. Blanchard JJ, Gangestad SW, Brown SA, Horan WP. Hedonic capacity and schizotypy revisited: a taxometric analysis of social anhedonia. J Abnorm Psychol 2000;109:87-95.

59. Bonfils KA, Lysaker PH, Minor KS, Salyers MP. Affective empathy in schizophrenia: a meta-analysis. Schizophr Res 2016;175:109-117. 